\title{
Personalised medicine comes a step closer for asthma
}

Scott Gottlieb New York

Researchers have discovered subtle differences in the DNA of people with asthma which help to predict which patients will benefit most from a certain drug before they take it, a new study has reported.

In tests of the DNA of 121 patients with asthma, researchers led by Dr Stephen Liggett, professor of medicine at the University of Cincinnati College of Medicine, and colleagues found four different patterns of DNA in a gene that helps to relax muscles in a person's lungs. Lung function was measured before and after treatment with salbutamol (known as albuterol in the United States), with the individual responses correlated to the sequence of variations found in discrete regions of a person's DNA.
Salbutamol, a drug that is aimed at spurring the muscle relaxing gene into greater action to treat asthmatic wheezing worked very well in those with one pattern, not at all in those with another, and moderately in the other two groups (Proceedings of the National Academy of Sciences 2000;97:10483-8).

It is the first example, researchers said, of personalised drug treatment, made possible by the sequencing of the human genome. In the future, the researchers said, they will be able to match individual patients to different medications, using their DNA as guidance to improve the drugs' effectiveness and eliminate harmful side effects. "Pharmacogenomics" is considered the most likely spin-off of decoding the human genome to have practical application in the near term.

Salbutamol works by blocking the $\beta_{2}$ adrenergic receptor. This causes muscles to relax and allows bronchial tubes to dilate, aiding the flow of air to the lungs. Identifying the genes that affect the way a person responds to the drug will help doctors to tailor prescriptions for each patient, said Dr Liggett.

"This is a step toward personalised medicine," said Dr Liggett. "The era of one drug fitting all is probably coming to an end soon."

The regions in DNA that the researchers were examining are single nucleotide polymorphisms. Some single nucleotide polymorphisms act like typographical errors, causing abnormalities in the DNA, while others do not seem to matter. In this case, researchers had previously found that the $\beta_{2}$ adrenergic receptor gene contains 13 of these sites where single nucleotide polymorphisms are present in the genetic code.

The scientists analysed all the possible differences people could have and found that four common differences predicted how people would respond to salbutamol.

Medicines now are generally tested on large numbers of people, with researchers looking at the average response of those taking the drug versus those taking placebo. "You'll see responses that are very, very good and some that are bad," Dr Liggett said. "The FDA says to look at the average response."

As a result, he said, some drugs that may be quite useful for a small subset of patients may never become available because they show little or no benefit, or they even harm, on the aggregate. By using examinations of DNA, researchers will be able to find subgroups of patients who benefit from certain drugs even if, on average, most patients show no response.

\section{Twelve die of West Nile virus in Israel}

Judy Siegel-Itzkovich Jerusalem

Twelve middle aged and elderly Israelis have died since August of complications of the mosquito borne disease West Nile virus, and an additional 157 have survived it. Between August and October 1999 in New York city more than 66 people were infected and six died.

The Israeli health ministry's associate director general, Dr Boaz Lev, had initially claimed that the high numbers were due to sophisticated laboratory tests first made available here last year that enabled the government to screen the blood of people complaining of symptoms.

As the numbers mounted, they conceded, however, that there was a genuine outbreak, and some experts in infectious diseases called it an epidemic. The West Nile virus is harmless in healthy people but can cause fatal inflammations of the brain and spinal cord in immune compromised or immune suppressed patients.

The youngest Israeli who died of the disease was a 54 year old woman who had myasthenia gravis; she was bitten by an infected mosquito and died of encephalitis. But most of the others were aged over 80 and had chronic diseases of old age.

The health ministry in Jerusalem was slow to wake up to the danger, apparently hoping in August that it would just blow over. This was despite the fact that New York health authorities had informed them last year that the viral strains they had isolated in the United States were closely similar to or identical with those found in Israel. They suggested that infected pet birds may have been smuggled from Israel to the United States and been responsible for the New York outbreak.

Panic is spreading, and hospital emergency rooms around the country are filling up with anxious healthy people bitten by mosquitoes or with mild flu-like symptoms.

Meanwhile, intensive pesticide spraying has been carried out at night, especially in the hardest hit area, around Sharon, north east of Tel Aviv. Environmentalists are worried that this "cure" might in the long term be worse than the disease itself.

As Israel is one of the world's major stopovers for migrating

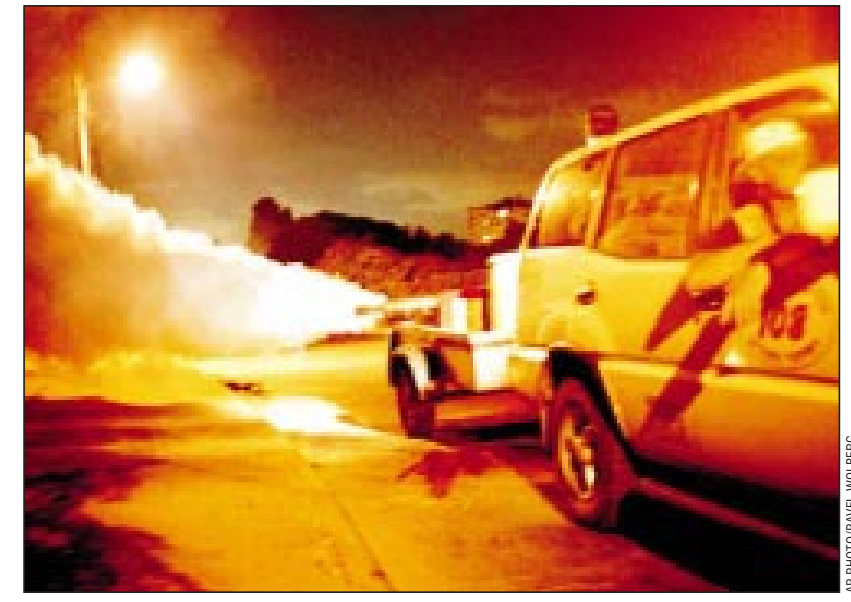

An Israeli municipal worker sprays anti-mosquito dust

birds in the autumn and spring, millions of them will be passing through nature reserves in the coming weeks, and some could be bearing West Nile virus.

Although Professor Manfred Green, head of the Israel Center for Disease Control, predicts that the outbreak will stop with the imminent colder weather and rainy season, others worry that Israel's winter temperatures might not be low enough to kill off all the mosquitoes.

Most Israelis have natural immunity to West Nile fever, which was first reported in Uganda in 1937; its ecology was char- acterised in Egypt in the 1950s, and an outbreak of meningoencephalitis (inflammation of the spinal cord and brain), affecting 400 people, was reported in Israel in 1957. It has since become endemic in the Middle East and Africa and has spread to Europe, central Asia, Oceania, and, most recently, North America.

Although mosquitoes bearing the flaviviridae family virus have usually contracted it by biting infected poultry (especially geese) and other birds, scientists have isolated the virus in mammals such as cats, dogs, and horses. 